\title{
Energy Conservation by Peer-to-Peer Relaying in Quasi-Ad Hoc Networks ${ }^{\star}$
}

\author{
Andrew Ka-Ho Leung and Yu-Kwong Kwok ${ }^{\star \star}$ \\ Department of Electrical and Electronic Engineering, \\ The University of Hong Kong, Pokfulam Road, Hong Kong \\ ykwok@hku.hk
}

\begin{abstract}
Thanks to the highly popular dual channel capabilities (e.g., GSM plus Bluetooth) in modern handheld personal communication devices, an integrated cellular and ad hoc peer-to-peer network (i.e., a quasi-ad hoc wireless network) has already been widely reckoned as a readily practicable and attractive mobile computing environment. In this paper, we propose a co-operative relaying scheme, called eeRelay, for such a quasi-ad hoc network, to extend the life-time of low energy level users significantly. More importantly, the energy efficiency of the whole network is also remarkably increased.
\end{abstract}

\section{Introduction}

The problem of short service life of most 3G handsets serves as an alarming alert that energy efficiency is a crucial factor in making a ubiquitous wireless communication system a successful story [7. In view of the fact that contemporary battery technology cannot solve the service life problem, in our study we find that "relaying" can be one promising strategy in addressing the issue.

Nowadays hand-held wireless communication devices are commonly equipped with more than one wireless interface and are capable of communicating both with the base station and directly with other users in ad hoc manner. Thus, we believe that an interesting kind of ubiquitous wireless networks in future is quasiad hoc in nature where communication links can be classified into two modes: (1) one-to-one communication among peers (e.g., a user shares files with another user directly using WLAN or data packets transmitted from one measurement node to another one) and (2) many-to-one communication from some nodes to the "sink" (e.g., mobile phone users access the base station in the uplink). We treat two modes of communication linkage differently (see Figure 1(a)

We propose a new collaborative energy management scheme, called eeRelay, for such a quasi-ad hoc network to increase the energy efficiency. We adapt the transmission power of mobile terminals to reduce the energy used. This is done by using another nearby higher energy level user as a relay.

The remainder of this paper is organized as follows. In Section 2, we provide some background information on relaying systems and describe our approach to

\footnotetext{
* This research was supported by a grant from the Research Grants Council of the HKSAR under project number HKU 7157/04E.

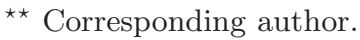


increasing the energy efficiency by relaying actions. In Section 3 , we present our simulation results. Finally, in Section 4 we discuss the benefit of our protocol by taking incentives into consideration.

\section{Related Work and Proposed Approach}

Relaying have been proposed in the literature [3], 5] and [9]. However, nearly all of these proposed relaying schemes do not explore the possibility of cooperation in energy domain. For this reason, we look at the relaying problem in another perspective in this paper. Specifically, we define who should be the relaying node so that the relaying process could be more energy efficient. We use the concept of "helpers" to replace the traditional simple concept of "relays".

\subsection{Design of the Proposed eeRelay Protocol}

We use cellular mobile network as an application example of quasi-ad hoc network to describe our energy efficient relaying protocol, namely eeRelay. When a low energy level user needs to set up a connection to the base station (BS). The BS dynamically select an energy efficient relay (helper) to relay the traffic for the low energy users. The BS is assumed to have location information of all users 1 In our design, a user is categorized as a "Helper" in Helper Set $\mathbf{H}$ if: (1) its energy level is high enough; and (2) it is physically situated in the "Helper's Region D" with respect to a low energy level user.

Mathematically, for a set of users $n_{1}, \ldots, n_{n} \in \mathbf{N}$, a user $n_{i} \in \mathbf{H}$ if $E\left(n_{i}\right) \geq$ $\gamma$ and $n_{i} \in \mathbf{D}$ where $E\left(n_{i}\right)$ denotes the energy level of a user $n_{i}, m \leq n, \mathbf{H} \subset \mathbf{N}$.

$\mathbf{D}$ is named as "Helpers' Region", and is defined as follows. It makes sure that, for a user to be qualified as a "Helper", it needs to be situated in certain geographical position such that the relationship $P_{u \rightarrow h}+P_{h \rightarrow B S}<P_{u \rightarrow B S}$ is satisfied. This ensures that the relaying action will not only reduce $u$ 's energy consumption but will also be an energy efficient one even when we focus on the total energy used.

We assume a path loss propagation model with path loss exponent $\beta$, i.e.,

$$
P_{A \rightarrow B}=k d_{A \rightarrow B}^{\beta}
$$

where $P_{A \rightarrow B}$ is the required transmit power between point $A$ and $B$ and $d_{A \rightarrow B}$ is the distance between them. In general, $\beta$ is an integral value ranges from two to four in urban areas. In our analysis we set it as four which is a value commonly used in the literature 8 . Finally, $k$ is a constant. 2

\footnotetext{
${ }^{1}$ The location information can be obtained by using GPS on mobile devices or techniques such as time-difference triangulation performed by three or more nearby base stations.

${ }^{2}$ Please note that the value of $\mathrm{k}$ could be different for communication links for $B S_{-}$ mobile terminal or mobile terminal-mobile terminal. But here we used one fixed value of $\mathrm{k}$ as an approximation. Our simulations use more realistic path loss model to show that this approximation is justified for the purpose of defining the Helper's Region.
} 


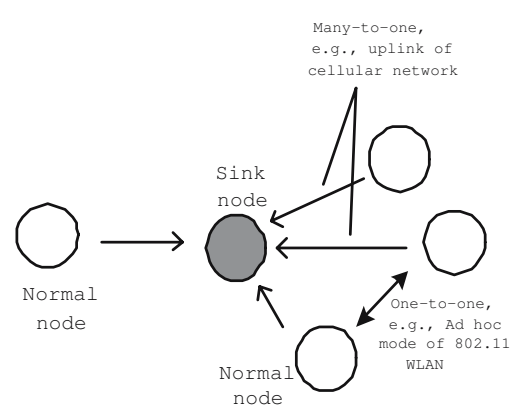

(a) A quasi-ad hoc network

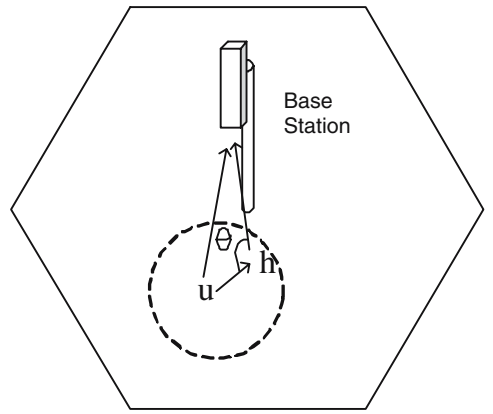

(b) Using a high energy "helper" to relay data for a low energy source

Fig. 1. eeRelay

Now let us consider Figure 1(b) in which $h$ acts as a relay of $u$ to communicate with the BS. Our goal is to find out under what circumstances would $P_{u \rightarrow h}+$ $P_{h \rightarrow B S}<P_{u \rightarrow B S}$ so that we can select a user (helper candidate) situated in such a position that its relaying action will not only reduce $u$ 's energy consumption but will also be an energy efficient one even when we focus on the total energy used.

According to our path loss model, to achieve this goal, we need:

$$
d_{u \rightarrow B S}^{4}>d_{u \rightarrow h}^{4}+d_{h \rightarrow B S}^{4}
$$

By cosine law, we have:

$$
d_{u \rightarrow B S^{2}}=d_{u \rightarrow h}{ }^{2}+d_{h \rightarrow B S^{2}}-2 d_{u \rightarrow h} d_{h \rightarrow B S} \cos \theta
$$

Substitute Equation (3) into Equation (2), we have:

$$
\Rightarrow \frac{2\left(d_{u \rightarrow h}{ }^{2}+d_{h \rightarrow B S}{ }^{2}\right) \cos \theta}{d_{u \rightarrow h} d_{h \rightarrow B S}}-2 \cos ^{2} \theta<1
$$

A Helper's Region with respect to the position of the lower energy usen 3 is plotted in Figure 2(f) (shaded area) and is found to be "ellipse-like".

\subsection{Implementation}

Phase I-Helper Request. At first, when a low power user $u$ wants to make a connection, it triggers the helper request process. It transmits, with extremely low power (say, $0.1 \mathrm{~W}$ or less), a "helper searching packet" to ask for any user nearby which could be a helper candidate according to the definition in Section 2.1 (see the condition specified by Equation (10). Any nearby user which

${ }^{3}$ In this paper the term "user" is used interchangeably with the term "UE" (User Terminal) used in conventional 3G UMTS-WCDMA system. 
receives this packet checks its energy level and see if it could be a helper candidate. If it fulfills the requirement of condition (1), it sends an "helper access packet" to BS on behalf of $u$, containing its own ID and $u$ 's ID after a random back off period so as to minimize the probability of all ACKs from different helper candidates colliding with each other. $u$ keeps a timer for "helper searching timeout". It re-sends a helper request packet if there is no reply from any nearby neighbor. If $u$ still fails in finding a helper after several attempts it will connect to BS on its own.

Phase II-Base Station Acknowledgment and Relay Set Up. Upon receiving the access packet from the helper candidate, the BS checks whether this helper candidate is inside the helper's region and confirms that the candidate's energy makes it eligible for helping $u$. BS collects helper access packets from all the helper candidates and form a helper set $\mathbf{H}_{\mathbf{u}}$ for $u$. BS then selects one eligible helper candidate $h$ from $\mathbf{H}_{\mathbf{u}}$ and acknowledges both $u$ and $h$ by sending an "BS-ACK packet" to them. After both $u$ and the helper candidate $h$ have received the BS-ACK, they do a handshaking with each other and connection from $u$ to BS through $h$ can be started.

Phase IV-Helper Maintenance and Tear Down. The helper set $\mathbf{H}_{\mathbf{u}}$ formed before is still useful after a helper $h$ has been selected for $u$ already. This is because both $u$ and $h$ are moving, there can be a case that $h$ is moving out of the "Helper's Region" of $u$ suddenly, or $h$ is moved to other cell, then a helper handoff is needed, so the BS asks the helper to update its position and $u$ 's position periodically during the connection ( $h$ can get $u$ 's GPS position information easily by asking $u$ to add it into the data packet periodically). Both $u$ and $h$ tear down the connection after $u$ terminates the call.

\section{Performance Results}

\subsection{Simulation Platform}

Now we study the performance of eeRelay using simulations. In our simulations, $50 \mathrm{UEs}$ are scattered randomly in an $800 \mathrm{~m} \times 800 \mathrm{~m}$ area initially and they are allowed to move freely according to the mobility model mentioned above. In this paper we consider a single cell case with no inter-cell handoff. We focus on our scheme and perform "helper handoff" only. The transmit power is selected when connection between BS and UE is established. We also assume that there is no collision in all ACK or BS-ACK transmissions. Each simulation is run for 50,000 seconds.

The wireless devices are assumed to have three possible modes of operation: Transmit, Receive and Idle. The energy consumption ratio of the three modes is set as $1: 0.6: 0.5$, as indicated by the experimental measurements done by Feeney and Nilsson [4]. The energy consumption on a node is modeled as

$$
P_{\mathrm{Tx}} T_{\mathrm{Tx}}+P_{\mathrm{Rx}} T_{\mathrm{Rx}}+P_{\mathrm{IDLE}} T_{\mathrm{IDLE}}
$$


where the first three $P$ terms represent power consumption in Transmit, Receive and Idle modes, respectively, the $T$ terms represent corresponding time durations that the mobile devices are in different modes.

Our scheme applies to the uplink of an integrated cellular and ad hoc network. To simulate this environment, we obtain reference system parameter values from the link budget for the uplink of a typical UMTS-WCDMA 3G system. The maximum transmit power of mobile node is $0.25 \mathrm{~W}(24 \mathrm{dBm})$ and the receiver Sensitivity for $384 \mathrm{kbps}$ data service at $\mathrm{BER}=10^{-3}$ is -109.2 dBm 6]: 4 Using the Okumura-Hata Model 6] we can estimate the transmission range of $\mathrm{BS}$ and mobile devices. We assume that the calls arrive according to a Poisson distribution where the call arrival times and the inter-arrival times between calls are mutually independent. Our mobility model assumes UEs' velocities follow a Gaussian distribution with mean $=3 \mathrm{~km} / \mathrm{hr}$, (i.e., $0.83 \mathrm{~m} / \mathrm{s}$ ) and variance $=0.54$.

\subsection{Simulation Results}

First we consider the amount of energy used for control purpose (e.g., transmission or reception of helper search packets, helper access packets, BS-ACK packets, relaying set up and torn down). As shown in Figure 2(e) the total amount of energy consumed for control purpose is below $0.2 \%$ of total energy of each node. As eeRelay is running, more calls are set up and tear down. After a transient period of around 5,000 seconds, the percentage of total energy consumed for control purpose falls and finally attains an approximately constant value.

Performance Metrics. We consider two performance metrics, the first one is for low energy level users defined according to Section 2.1. We define the performance metric as "Percentage life extension of $u_{k}$ ":

$$
\frac{\text { life of } u_{k} \text { with helper scheme }- \text { life of } u_{k} \text { without helper scheme }}{\text { life of } u_{k} \text { without helper scheme }} \times 100
$$

where $u_{k} \in \mathbf{U}, k \leq n$. Here, "life" is defined as the total duration of time that a UE operates until it exhausts its battery. From this metric we define the average percentage life extension of low energy level users as:

$\frac{\sum_{k} \quad \text { Percentage life extension of } u_{k}}{k}$

A similar metric, which sums and averages over all users is defined as a measure for the overall energy efficiency for the whole network, namely "Percentage life extension of all users".

4 "UE" and "Node B" are the terminology used in UMTS-WCDMA specification for Mobile Users and Base Station. We follow this convention and use each pair of them interchangeably in this paper. 

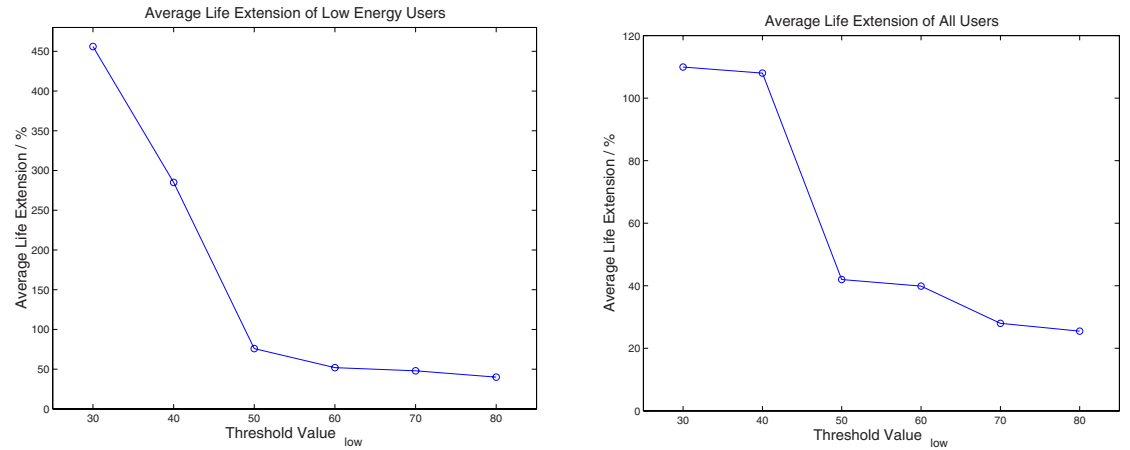

(a) Average life extension of low energy (b) Average life extension of all users, level users, mean velocity $=0.83 \mathrm{~m} / \mathrm{s}$ mean velocity $=0.83 \mathrm{~m} / \mathrm{s}$
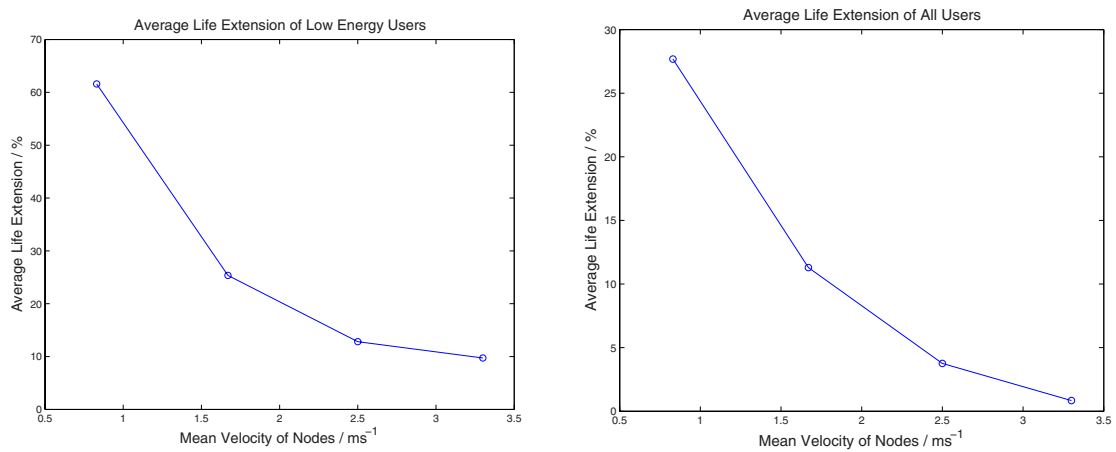

(c) Average life extension of low energy (d) Average life extension of all users, $\gamma$ level users, $\gamma=50$ $=50$
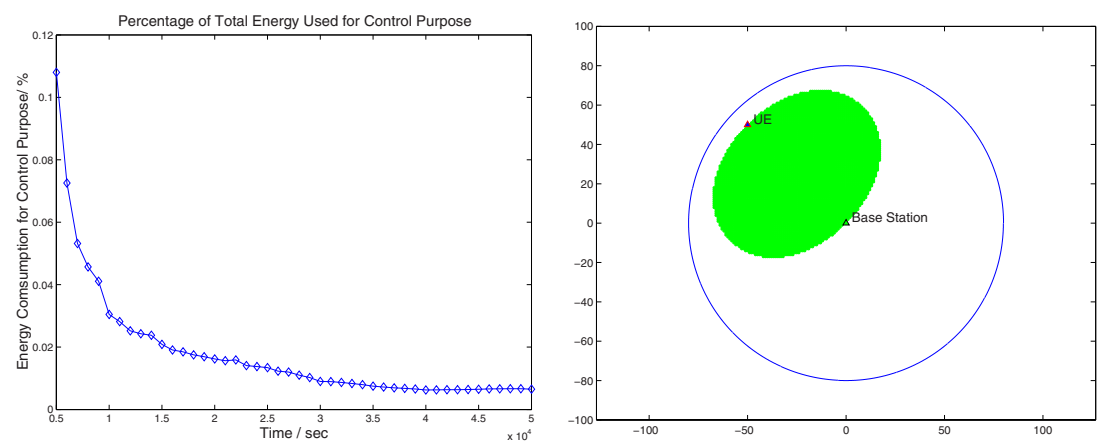

(e) Percentage of total energy consumed (f) Helper's Region of a UE at $(-50,50)$ for control purpose, $\gamma=50$, mean veloc- ; BS at $(0,0)$ ity $=0.83 \mathrm{~m} / \mathrm{s}$

Fig. 2. Protocol performance

Effect of Threshold $\gamma$. For a mean velocity of users $=0.83 \mathrm{~m} / \mathrm{s}, \epsilon=0.5$, the performance metrics we defined are plotted in Figure 2(a) and 2(b). We find that our scheme yields significant average life extension on the low energy 
users $u_{k}$, ranging from $456 \%$ to $40 \%$ for $\gamma$ ranging from 30 to 80 , depending on the threshold value $\gamma$ (i.e., the definition of "low energy level user"). If the threshold is set to be very low (e.g., 30), then the average life extension for those low energy level users $u_{k}$ is very significant (over 400\%). We believe that this is due to the contribution from a large number of helpers (e.g., 38 helpers out of 50 users for $\gamma=30$ in the example we plot). But the tradeoff is the number of users that can be classified as "low energy level user" is small and thus the ratio of users who can gain benefit is small (12 low energy level users out of 50 users). A more fair scheme is to define the value of $\gamma$ as half of the maximum value of energy levels, the number of $u$ and $h$ would then be half-half, assume that the energy levels of all UEs are uniformly distributed. This "half-half" case yields an average life extension of around $76 \%$. The simulation results also show increases in life extension averaged over all users, representing a system-wide energy efficiency. The life extension ranges from around $110 \%$ to around $28 \%$ (see Figure 2(b) . To conclude, we see a tradeoff between the average gains by each of the low energy level user $\left(u_{k}, k \leq n\right)$ and the total number of low energy level users who could gain $(k)$.

Effect of Mobility of Users. For $\gamma=50$ (the numbers of low energy level users and helpers are half-half), the performance metrics we defined are plotted against mean velocity of users in Figures 2(c) and 2(d)

From our simulation results, we find that the gain through the use of our scheme is sensitive to the mobility of users. Consider Figures 2(c) and 2(d) that when the mean velocity of users increases from $0.83 \mathrm{~m} / \mathrm{s}(3 \mathrm{~km} /$ hour $)$ to $2.5 \mathrm{~m} / \mathrm{s}$ (12 km/hour), the average life extension of low energy level users decreases from around $61.6 \%$ to around $9.7 \%$ while the life extension averaged over all users also decreases from $27.6 \%$ to around $0.8 \%$ as the mobility increases from 0.83 $\mathrm{m} / \mathrm{s}$ to $2.5 \mathrm{~m} / \mathrm{s}$. This shows that our scheme is more suitable for low mobility users (e.g., pedestrians or people walking around in a shopping mall).

\section{Incentives}

Our scheme can be considered as "beneficial" on two aspects: (i) energy consumption of the whole network is reduced; and (ii) energy consumption of low energy level users is also reduced. Since helpers are asked to "share" their energy among those who need, this causes extra energy consumption on them, you may ask: Are those helpers in fact "losers" in our protocol? The answer is no and the reasons are: (1) when every node is running eeRelay protocol, it guarantees that whenever any node is in low energy state, high energy level users in its helper's region would help it by relaying its traffic, this provides incentive for any node to act as a helper when it is in high energy state since other nodes would help it in return when its energy level falls below the threshold; (2) In application like P2P file sharing, extending the life of low energy level users means more nodes could contribute to the P2P community. These arguments justify as to why we need to help those who are at low energy levels. 


\section{Conclusions}

We have presented a new energy sharing scheme. With our theoretical analysis and simulations, we also demonstrate the increased overall energy efficiency. We believe that the key point to extend the service life of mobile user terminals is the co-operation between users.

\section{References}

1. P. Agrawal, "Energy efficient protocols for wireless systems," Proc. IEEE PIMRC 1998, vol. 2, pp. 564-569, Sept. 1998.

2. BitTorrent, http://bitconjurer.org/BitTorrent/, 2004.

3. S. Doshi, S. Bhandare and T. X Brown, "An On-demand minimum energy routing protocol for a wireless ad hoc network," ACM SIGMOBILE Mobile Computing and Communications Review, vol. 6, no. 2, pp 50-66, July 2002.

4. L. M. Feeney and M. Nilsson, "Investigating the energy consumption of a wireless network interface in an ad hoc networking environment," Proc. IEEE INFOCOM 2001, vol. 3, pp. 1548-1557, Apr. 2001.

5. T. J. Harrold and A. R. Nix, "Intelligent relaying for future personal communication systems," Proc. IEE Colloquium on Capacity and Range Enhancement Techniques for the Third Generation Mobile Comm. and Beyond (Ref. No. 2000/003), Feb. 2000.

6. H. Holma and A. Toskala, WCDMA for UMTS: radio access for third generation mobile communications, John Wiley \& Sons, 2002.

7. C. Panasik, "Getting the most from 3G: battery life is key," available from http://www.findarticles.com/p/articles/mi_mOMLY/is_9_3/ai_108331198, 2004

8. T. S. Rappaport, Wireless communication, principle and practice, Prentice Hall, 1996.

9. H. Wu, C. Qiao, S. De and O. Tonguz, "Integrated cellular and ad hoc relaying systems: iCAR," IEEE Journal on Selected Areas in Comm., vol. 19, no. 10, pp. 2105-2115, Oct. 2001. 